\title{
Uncertain opinion formation based on the bounded confidence model ${ }^{*}$
}

\author{
Haiming Liang, Yucheng Dong, Cong-Cong Li \\ Business School, Sichuan University, Chengdu 610065, China
}

\begin{abstract}
Opinion formation is well used to investigate a consensus or several clusters among the opinions of a group of interaction agents. This study proposes several bounded confidence models to discuss the uncertain opinion formation. In the proposed models, the agents' various tolerances (zero-tolerance, partial tolerance and complete tolerance) on the uncertain opinions are firstly identified. Then, the relevant communication regimes are given to determine the confidence set, and the updated opinions are further calculated. Finally, we explore the influences of various types of agents and self-support on the average number of clusters through simulation analysis.
\end{abstract}

Keywords: Opinion formation, Uncertain opinion, Consensus, Tolerance, Bounded confidence model, Communication regime

\section{Introduction}

Opinion formation can be defined as a dynamic and interactive process. In opinion formation, a group of interaction agents represent their own initial opinions (evaluations) about a same issue, and based on some interaction rules, their opinions are continuously updated as the time step increases. And a consensus or a certain cluster among the opinion$\mathrm{s}$ is finally obtained. Previous studies have shown that opinion formation plays an important role in modeling and predicting the observations of group of agents on the practical issue [1]. Thus, opinion formation, especially the discussion on reaching a consensus $[2,3,4]$ has received increasing attention in the field of decision analysis.

Some research works on opinion formation models have been conducted, such as voter model [5], persuasiveness and supportiveness model [6], bounded confidence model [7, 8] and Alexford model [9]. In these existing models, using the bounded confidence model to solve opinion formation has become one of the hot research topics in recent years.

The bounded confidence model supposes individuals are willing to communicate with peers who have similar opinions and tend to ignore peers with sufficiently different opinions [10]. The earliest research result on bounded confidence model was p-

\footnotetext{
${ }^{*}$ This work was supported by a grant (No. 71171160) from NSF of China.
}

resented by Deffuant and Weisbuch (DW model)[7], and Hegselmann and Krause (2002) (HK model) [8]. Based on the DW model and HK model, several studies on bounded confidence model have been founded: (i) The discrete opinion dynamics model with the homogeneous bounded confidence [11, 12]; (ii) The discrete opinion dynamics model with the heterogeneous bounded confidence [13, 14]; (iii) The continuous opinion dynamics model with the homogenous bounded confidence $[15,16]$; (iv) The continuous opinion dynamics model with the heterogeneous bounded confidence [13, 17].

Previous studies have significant contributions to opinion formation. In these studies, the bounded confidence models are well used to determine the confidence set, calculate the weight of agents and update the opinions. In this study, we will extend the bounded confidence model to discuss the uncertain opinion formation. Compared with the previous studies on opinion formation, our proposal can mainly fill two gaps:

(1) The existing researches on opinion formation, such as [1] and [10], mainly focus on the crisp opinion represented by the agents. In practical opinion formation problem, due to the limitation of knowledge and experience, it is common that some agents will use uncertain opinion (often interval numbers) to represent their fuzzy thinkings[18, 19].

(2) When representing crisp opinions, the agents can directly identify their confidence sets and represent their updated opinions by referencing other agents' opinions. However, in the process of uncertain opinion formation, some agents will often provide some individualized tolerances on the uncertainty of their future opinions. For example, someone will hope that their representations are crisp opinions all the time, but others will be regardless of the uncertainty of their future opinions. Motivated by this consideration, this study proposes three bounded confidence models for different types of agents.

In order to do so, the rest of paper is organized as follows. Section 2 provides a brief introduction on the HK model and a notation description on our proposal. Then, three bounded confidence models considering the agents' tolerances on the uncertainty of opinions are proposed in Section 3. In Section 4, we explore the influence of number of agents and self-support on the average number of clusters through simulation analysis. Finally, concluding remarks are presented in Section 5. 


\section{Preliminary and the research problem}

The fundamental difference between the two classical bounded confidence models (DW and HK model$\mathrm{s}$ ) is the number of agents that communicate, which labels as the communication regime. In this paper, without loss of generality, we adopt the original HK model as the based model. If we adopt the original DW model as the based model, a similar study can be proposed. Therefore, in this section, we briefly introduce the original HK model [8] and the research problem, which are providing a well-grounded basis for the proposed model.

\subsection{Preliminary: the HK model}

Consider a population of $N$ agents, represented in a set $A=\left\{A_{1}, A_{2}, \ldots, A_{N}\right\}$. A set of discrete time $T=\{0,1, \ldots, M\}$ is used to model the repeated process of opinion process, where $M$ is a big enough integer number. Each agent $A_{i}$ at time $t$ has continuously varying opinion as $x_{i}(t)$ between zero and one, and $X(t)$ is the vector of opinions of all agents at time $t$ called opinion profile. Let $\varepsilon$ be the homogeneous bounded confidence of all the agents, where the agent only considers the opinions which differ not more than $\varepsilon$ from his/her opinion.

The process of solving the opinion dynamics problem by means of the HK model [8] includes three steps:

(1) Determination of the confidence set. Let $I\left(A_{i}, x_{i}(t)\right)$ be the confidence set of agent $A_{i}$ at time $t$. For any agent $A_{j}$, if $A_{j} \in I\left(A_{i}, x_{i}(t)\right)$, then the opinion $x_{j}(t)$ is considered by the agent $A_{i} . I\left(A_{i}, x_{i}(t)\right)$ is determined by

$$
I\left(A_{i}, x_{i}(t)\right)=\left\{A_{j} \in A|| x_{i}(t)-x_{j}(t) \mid<\varepsilon\right\}
$$

(2) Calculation of the weight. Let $w_{i j}(t)$ be the weight of agent $A_{i}$ assigns to agent $A_{j}, w_{i j}(t) \geq$ $0, \sum_{j=1}^{N} w_{i j}(t)=1$. Using the confidence set $I\left(A_{i}, x_{i}(t)\right)$, we can calculate the weight $w_{i j}(t)$, i.e.,

$$
w_{i j}(t)=\left\{\begin{array}{cc}
1 /\left|I\left(A_{i}, X(t)\right)\right|, & A_{j} \in I\left(A_{i}, X(t)\right) \\
0, & A_{j} \notin I\left(A_{i}, X(t)\right)
\end{array}\right.
$$

(3) Evolution of the opinions. The evolution of the opinions in the original HK model is modeled as a weighted arithmetic mean of opinions in the confidence set, i.e.,

$$
x_{i}(t+1)=\sum_{j=1}^{N} w_{i j}(t) x_{j}(t)
$$

\subsection{The research problem}

Different from only the crisp representations of agents in the existing model, we consider the case that the agents present both the crisp and uncertain opinions in this paper. Therefore, the opinions of $x_{i}(t)$ can be described as follows:(1) Crisp opinion. $0 \leq x_{i}(t) \leq 1$; (2) Uncertain opinion. $x_{i}(t)=\left[x_{i}^{L}(t), x_{i}^{U}(t)\right], 0 \leq x_{i}^{L}(t) \leq x_{i}^{U}(t) \leq 1$.
For notation simplicity, let $A^{C}$ and $A^{I}$ be two subsets of $A$, representing the agents whose initial opinions are presented as crisp and uncertain opinions, respectively, $A^{C} \cup A^{I}=A$ and $A^{C} \cap A^{I}=\emptyset$.

Furthermore, we also consider the agents' tolerances on the uncertainty of opinions. The notations on three types of agents are further described as: (1) zero tolerance agent set $A^{C 1}$ : the set of agents who can't tolerate the uncertainty of opinion all the time; (2) partial tolerance agent set $A^{I 1}$ : the set of agents who can tolerate their own uncertain opinion but can't tolerate the uncertainty of opinion of others; (3) complete tolerance agent set $A^{I 2}$ and $A^{C 2}$ : the set of agents in $A^{C}$ and $A^{I}$ who are regardless of the uncertainty of opinions all the time.

Based on the above notations, the aim of our proposal is to how to propose the new bounded confidence models to discuss the uncertain opinion formation.

\section{The proposed model}

In this section, inspired by the original HK model, we propose the new bounded confidence models, in which the agents' tolerances on the uncertainty of opinions are considered.

\subsection{Determine the confidence set}

Let $d\left(x_{i}(t), x_{j}(t)\right)$ be the difference between the opinions of agents $A_{i}$ and $A_{j}$ at time $t$, where

$$
d\left(x_{i}(t), x_{j}(t)\right)=\sqrt{\frac{\left(x_{i}^{L}(t)-x_{j}^{L}(t)\right)^{2}+\left(x_{i}^{U}(t)-x_{j}^{U}(t)\right)^{2}}{2}}
$$

The confidence set of agents can be determined by dividing into the following two cases.

Case A. For the agents in the set $A^{C 1}$ and $A^{I 1}$, when communicating with the agent in the $A^{I}$, the communication regime can be described by the following processes.

(1) Communication. It refers to that the agent $A_{i}$ will communicate with all the agents in $A^{I}$;

(2) Selective estimation. If $d\left(x_{i}(t), x_{j}(t)\right)<\varepsilon$, then agent $A_{i}$ will estimate a crisp opinion $f_{i j}(t)$ on the uncertain opinion of agent $A_{j}$. Otherwise, agent $A_{i}$ will discard the opinion $x_{j}(t)$. Here $f_{i j}(t) \in$ $\left[x_{j}^{L}(t), x_{j}^{U}(t)\right]$.

(3) Selective adoption. If $d\left(x_{i}(t), f_{i j}(t)\right)<\varepsilon$, then agent $A_{i}$ will adopt the opinion $f_{i j}(t)$. Otherwise, agent $A_{i}$ will discard the opinion $f_{i j}(t)$.

Case B. For the communication regime among agents except case $\mathrm{A}$, only the process of communication and selective adoption is considered. If $d\left(x_{i}(t), x_{j}(t)\right)<\varepsilon$, agent $A_{i}$ will directly adopt the crisp or uncertain opinion $x_{j}(t)$.

Based on cases A and B,

$$
I\left(A_{i}, X(t)\right)=\left\{\begin{array}{cc}
I_{1} \cup I_{2}, & A_{i} \in A^{I 1} \cup A^{C 1} \\
I_{3}, & \text { otherwise }
\end{array}\right.
$$

where $I_{1}=\left\{A_{j} \in A^{I 1} \mid d\left(x_{i}(t), f_{i j}(t)\right) \leq \varepsilon\right\}, I_{2}=\{$ $\left.A_{j} \in A^{C 1} \mid d\left(x_{i}(t), x_{j}(t)\right) \leq \varepsilon\right\}$ and $I_{3}=\left\{A_{j} \in A \mid d(\right.$ $\left.\left.x_{i}(t), x_{j}(t)\right) \leq \varepsilon\right\}, d\left(x_{i}(t), x_{j}(t)\right)$ and $d\left(x_{i}(t), f_{i j}(t)\right)$ 
in case $\mathrm{A}$ and case B are determined by using the distance measure for interval numbers and crisp numbers, respectively.

\subsection{Obtain the updated opinions}

After obtaining the confidence set $I\left(A_{i}, x_{i}(t)\right)$, the weight $w_{i j}(t)$ can also be determined by using Eq. (2). Likewise, the updated opinions are modeled as a weighted arithmetic mean of opinions in the confidence set. Since Urbig et al. [12] have stated that an agent may assign a weight of $\mu$ to the own opinion in opinion dynamics, we also incorporate the self-support $\mu$ into our evolution of the opinions.

For $A_{i} \in A^{C 1}$, the new opinions $x_{i}(t+1)$ can be determined as:

$$
\begin{aligned}
& x_{i}(t+1)=\mu x_{i}(t)+(1-\mu)\left(\sum_{A_{j} \in I\left(A_{i}, X(t)\right) \cap A^{C}}\right. \\
& \left.w_{i j}(t) x_{j}(t)+\sum_{A_{j} \in I\left(A_{i}, X(t)\right) \cap A^{I}} w_{i j}(t) f_{i j}(t)\right)
\end{aligned}
$$

For $A_{i} \in A^{I 1}$, the new opinions $x_{i}(t+1)=\left[x_{i}^{L}(t+\right.$ 1), $\left.x_{i}^{U}(t+1)\right]$ can be determined as:

$$
\begin{array}{r}
x_{i}^{L}(t+1)=\mu x_{i}^{L}(t)+(1-\mu)\left(\sum_{A_{j} \in I\left(A_{i}, X(t)\right) \cap A^{C}} w_{i j}(t) f_{i j}(t)\right) \\
w_{i j}(t) x_{j}(t)+\sum_{A_{j} \in I\left(A_{i}, X(t)\right) \cap A^{I}} \\
x_{i}^{U}(t+1)=\mu x_{i}^{U}(t)+(1-\mu)\left(\sum_{A_{j} \in I\left(A_{i}, X(t)\right) \cap A^{C}} w_{i j}(t) f_{i j}(t)\right) \\
w_{i j}(t) x_{j}(t)+\sum_{A_{j} \in I\left(A_{i}, X(t)\right) \cap A^{I}}
\end{array}
$$

For $A_{i} \in A^{C 2} \cup A^{I 2}$, the updated opinions $x_{i}(t+$ $1)=\left[x_{i}^{L}(t+1), x_{i}^{U}(t+1)\right]$ can be determined as:

$$
\begin{array}{r}
x_{i}^{L}(t+1)=\mu x_{i}^{L}(t)+(1-\mu) \sum_{A_{j} \in I\left(A_{i}, X(t)\right)} \\
w_{i j}(t) x_{j}^{L}(t) \\
x_{i}^{U}(t+1)=\mu x_{i}^{L}(t)+(1-\mu) \sum_{A_{j} \in I\left(A_{i}, X(t)\right)} \\
w_{i j}(t) x_{j}^{U}(t)
\end{array}
$$

\section{Simulation analysis}

In this section, through simulation experiments, we mainly investigate the influences of two factors on the average numbers of clusters, i.e., the number of agents in the sets $A^{C 1}, A^{I 1}, A^{C 2}$ and $A^{I 2}$, and the self-support.

\subsection{The influences of the number of agents}

Let $\alpha^{C 1}, \alpha^{I 1}, \alpha^{C 2}$ and $\alpha^{I 2}$ be the relevant ratios of the number of agents in the sets $A^{C 1}, A^{I 1}, A^{C 2}$ and $A^{I 2}$, respectively, $\alpha^{C 1}+\alpha^{I 1}+\alpha^{C 2}+\alpha^{I 2}=1$, $\alpha^{C 1}, \alpha^{I 1}, \alpha^{C 2}, \alpha^{I 2} \geq 0$. In the following, the influences of ratios $\alpha^{C 1}$ and $\alpha^{I 1}$ are first analyzed.

We study here the populations of 500, 1000, 1500 and 2000 agents and vary the values of $\alpha^{C 1}$ and $\alpha^{I 1}$, while keeping the self support $\mu=0, \alpha^{C 2}=0$ and $\alpha^{I 2}=0$. When setting different values of $\alpha^{C 1}$ and $\alpha^{I 1}\left(\alpha^{C 1}+\alpha^{I 1}=1\right)$, we respectively run 200 times simulation to obtain the average number of clusters. Figure 1 plots the average number of clusters with different $\alpha^{C 1}$, population sizes and the bounded confidences $\varepsilon=0.1, \varepsilon=0.15$ and $\varepsilon=0.2$.

$$
\varepsilon=0.1
$$

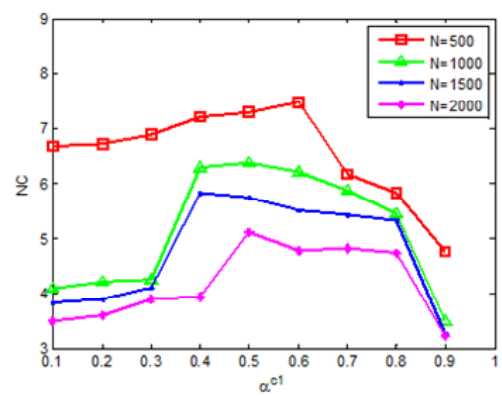

$\varepsilon=0.15$

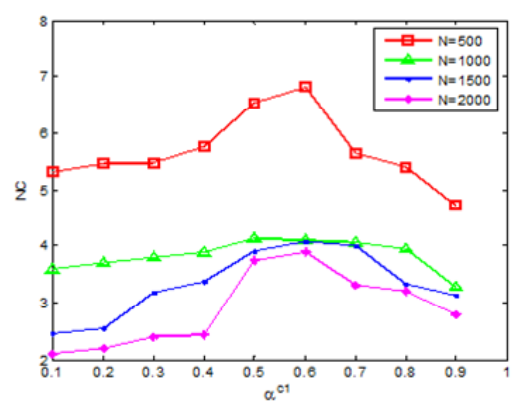

$\varepsilon=0.2$

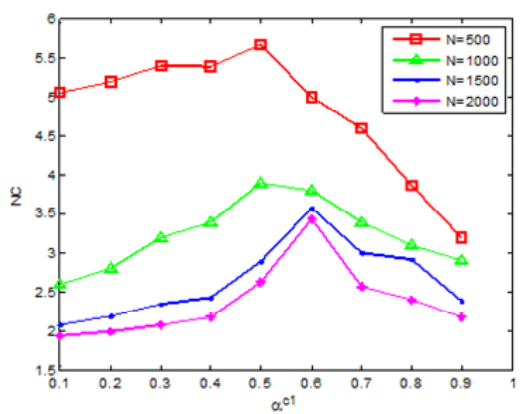

Figure 1: The average number of clusters with different ratios $\alpha^{C 1}$.

From Figure 1, we have the following observations:

(1) The number of clusters increases for an increasing ratio $\alpha^{C 1}$ and an decreasing ratio $\alpha^{I 1}$ while about a specific threshold it decreases again. 
(2) In this case for only involving the agents in the sets $A^{C 1}$ and $A^{I 1}$, the number of clusters decreases as the bounded confidence increases. And the larger the number of agents is, the smaller the number of clusters is.

Also suppose that $\alpha^{C 1}=0$ and $\alpha^{I 1}=0$, we explore the influence of ratios $\alpha^{C 2}$ and $\alpha^{I 2}$ on the average number of clusters. Figure 2 plots the average number of clusters with different $\alpha^{C 2}$, population sizes and the bounded confidences $\varepsilon=0.1, \quad \varepsilon=0.15$ and $\varepsilon=0.2$.

$$
\varepsilon=0.1
$$

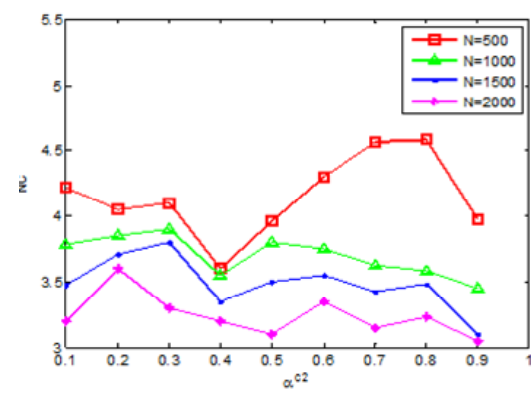

$\varepsilon=0.15$

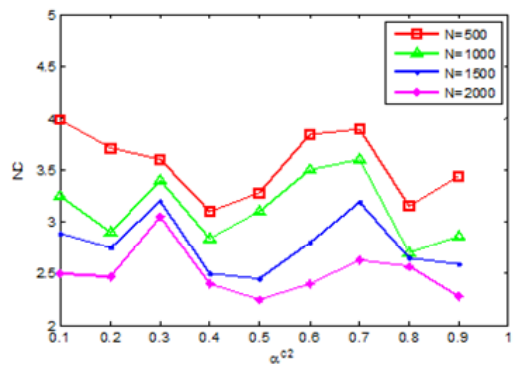

$\varepsilon=0.2$

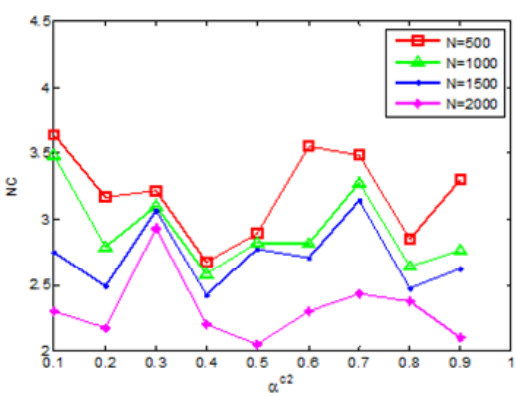

Figure 2: The average number of clusters under different ratios $\alpha^{C 2}$.

From Figure 2, we have the following observations:

(1) The average number of clusters fluctuates for an increasing ratio $\alpha^{C 2}$ and an decreasing ratio $\alpha^{I 2}$. Meanwhile, the average number of clusters with the ratios $\alpha^{C 2}$ and $\alpha^{I 2}$ is significantly smaller than that in the same ratios $\alpha^{C 1}$ and $\alpha^{I 1}$.
(2) In this case for only involving the agents in the sets $A^{C 2}$ and $A^{I 2}$, the number of clusters decreases as the bounded confidence increases. And the larger the number of agents is, the smaller the number of clusters is.

Further, let $\alpha^{C}$ and $\alpha^{I}$ be the relevant ratios of agents in the sets $A^{C}$ and $A^{I}$, respectively, $\alpha^{C}=$ $\alpha^{C 1}+\alpha^{C 2}$ and $\alpha^{I}=\alpha^{I 1}+\alpha^{I 2}$. Suppose that $\alpha^{C 1}=\alpha^{C 2}$ and $\alpha^{I 1}=\alpha^{I 2}$, we explore the influence of ratios $\alpha^{C}$ and $\alpha^{I}$ on the average number of clusters. Figure 3 plots the average number of clusters with different $\alpha^{C}$, population sizes and the bounded confidences $\varepsilon=0.1, \varepsilon=0.15$, and $\varepsilon=0.2$.

$$
\varepsilon=0.1
$$

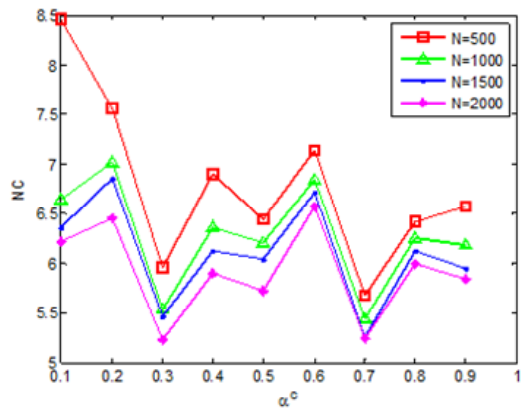

$\varepsilon=0.15$

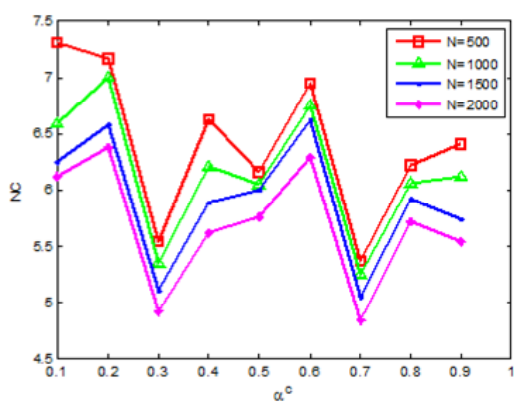

$\varepsilon=0.2$

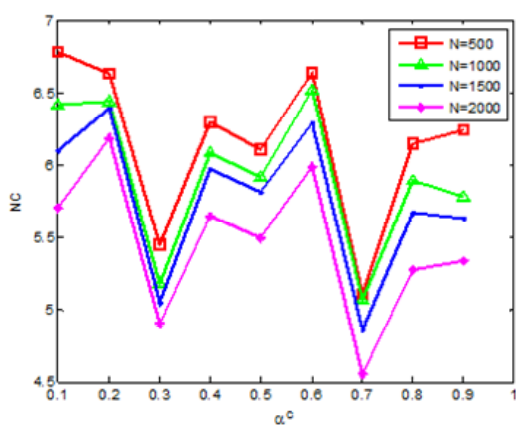

Figure 3: The average number of clusters with different ratios $\alpha^{C}$.

From Figure 3, we have the following observations:

(1) The average number of clusters fluctuates for an increasing ratio $\alpha^{C}$ and an decreasing ratio $\alpha^{I}$. 
This can be attributed to the role of ratios $\alpha^{C 2}$ and $\alpha^{I 2}$.

(2) The more average number of clusters is obtained under the existence of four types of agents. Also, the larger the number of agents, the smaller the number of clusters.

\subsection{The influences of the self-support}

Consider an example with a fixed initial opinion profile $X(0)$ of 500 agents, $\varepsilon=0.05$, and three stages for the parameter $\mu$.

$$
\varepsilon=0.05, \mu=0
$$

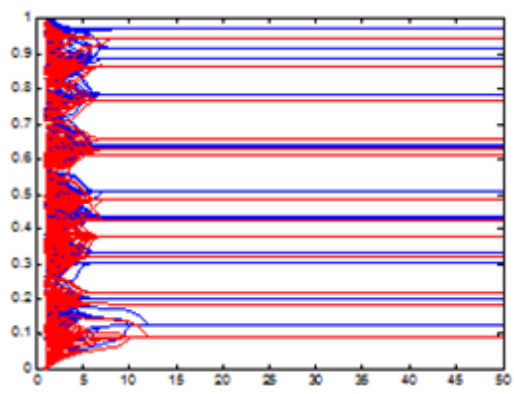

$\varepsilon=0.05, \mu=0.35$

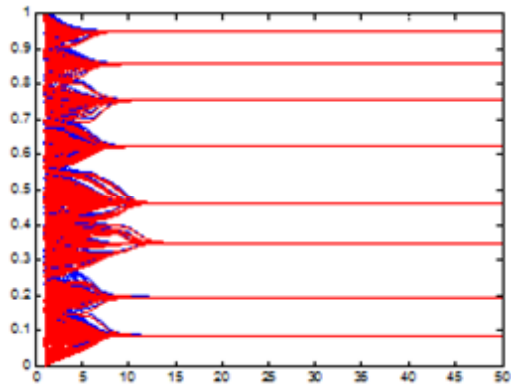

$\varepsilon=0.05, \mu=0.75$

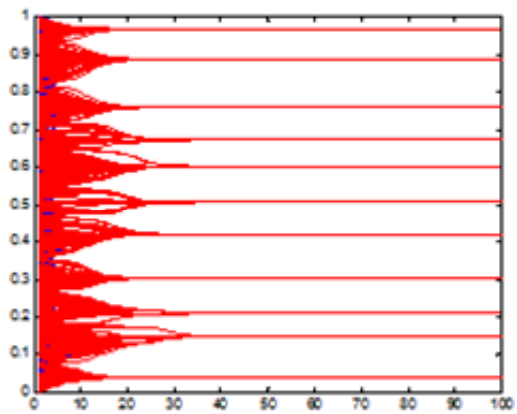

Figure 4: Example for one opinion profile $X(0)$, $\varepsilon=0.05$ and $\mu=0,0.35,0.75$.

Figure 4 plots the variation of the number of clusters. And the red and blue lines in Figure 4 denote the evolution of upper and lower bounds of uncertain opinions, respectively. From Figure 4, we can see that increasing $\mu$ from 0 to 0.35 take the number of clusters from 13 to 8 , but increasing $\mu$ further from 0.35 to 0.75 take the number of clusters from 8 to 11 .

In the following, we consider 200 fixed initial opinion profiles of 500 agents for $\mu, \varepsilon=0.1$, and nine stages for the parameter $\mu$. By setting different values of $\alpha^{C 1}\left(\alpha^{I 1}=1-\alpha^{C 1}\right), \alpha^{C 2}\left(\alpha^{I 2}=1-\alpha^{C 2}\right)$ and $\left(\alpha^{C 1}=\alpha^{C 2}\right.$ and $\left.\alpha^{I 1}=\alpha^{I 2}\right)$, we explore the influence of $\mu$ and $\alpha^{C 1}, \alpha^{C 2}, \alpha^{C}$ on the average number of clusters, respectively.
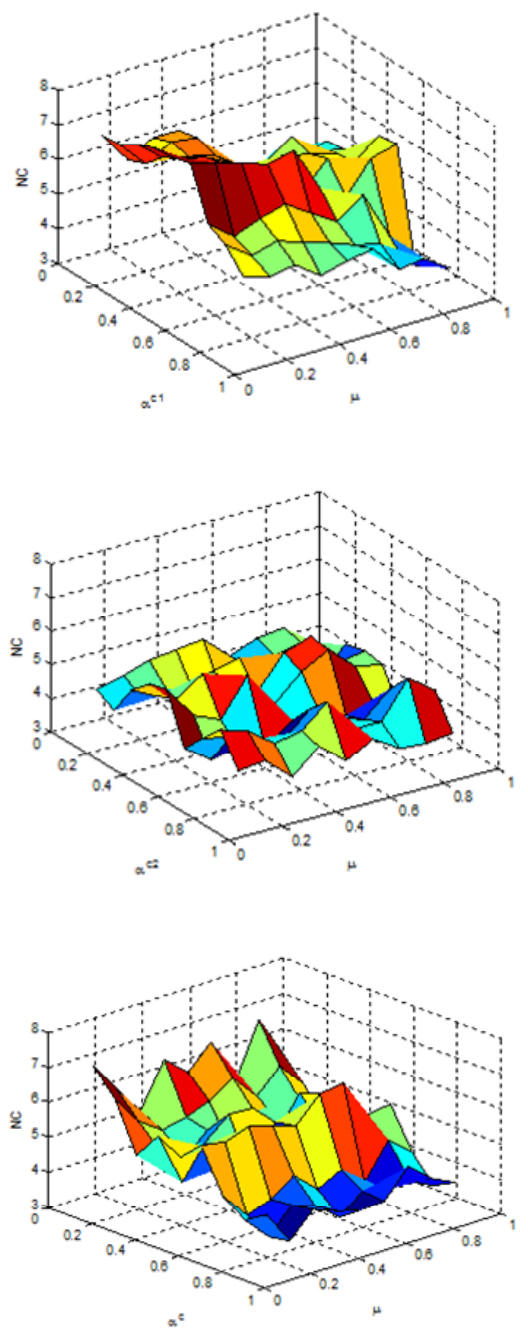

Figure 5: The average number of clusters under different ratios $\alpha^{C 1}, \alpha^{C 2}, \alpha^{C}$ and self-supports $\mu$

From Figure 5, we obtain the following observations:

(1) The average number of clusters fluctuates for an increasing ratio $\alpha^{C 1}$ and an increasing selfsupport $\mu$. And the similar results are obtained by increasing $\alpha^{C 2}$ and $\alpha^{C}$, respectively.

(2) The variations for the average number of clusters is smallest when setting different values of $\alpha^{C 2}$ and $\mu$, and the variations for the average number of clusters is biggest when setting different values of $\alpha^{C}$ and $\mu$.

Then, we explore the influence of $\mu$ and different population size $N$ on the average number of clusters. 
We study here the populations of 500, 1000, 1500 and 2000 agents and varying $\mu$ from 0.1 to 0.9 , while keeping $\alpha^{C 1}=\alpha^{C 2}=\alpha^{C}=0.5$. We use the fixed $N$ initial opinions when varying $\mu$ in one simulation. We singly run the simulation 200 times.

$$
\alpha^{C 1}=0.5
$$

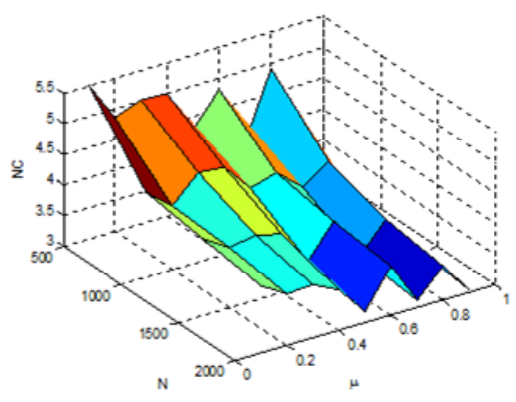

$$
\alpha^{C 2}=0.5
$$

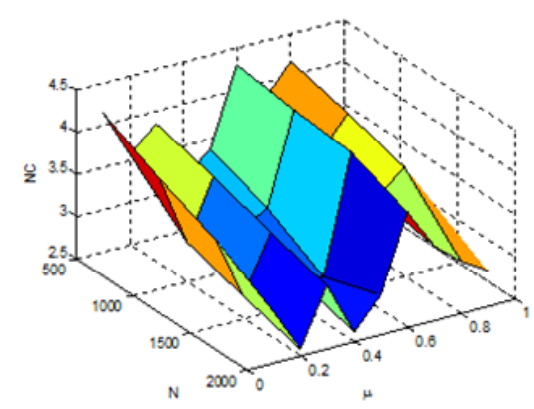

$$
\alpha^{C}=0.5
$$

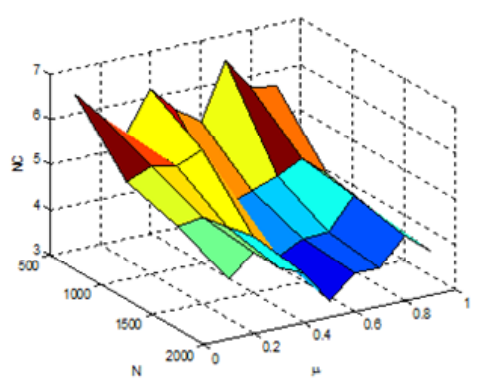

Figure 6: The average number of clusters with different population sizes $N$ and self-supports $\mu$

From Figure 6, we have the following observations:

(1) The average number of clusters fluctuates for a fixed ratio $\alpha^{C 1}$, a fixed population size $N$ and an increasing self-support $\mu$. And the similar results are obtained under a fixed ratio $\alpha^{C 2}$ and $\alpha^{C}$, respectively.

(2) The average number of clusters decreases for a fixed ratio $\alpha^{C 1}$, a fixed self-support $\mu$ and an increasing population size $N$. And the similar results are obtained under a fixed ratio $\alpha^{C 2}$ and $\alpha^{C}$, respectively.

\section{Conclusions}

This paper discusses the uncertain opinion formation based on the bounded confidence model. The main contributions presented are as follows:

(1) The uncertain opinions presented by some agents are incorporated into the process of opinion formation. Furthermore, we also consider the agents' various tolerances (zero-tolerance, partial tolerance and complete tolerance) on the uncertainty of opinions to discuss the uncertain opinion formation.

(2) According to the agents' various tolerances and their providing opinions, the relevant communication regimes are given to determine the confidence set. And the weights of agents assigned by each agent is determined. Further, several bounded confidence models are proposed.

(3) We design the simulation experiments to explore the influences of the ratios of agents and selfsupport on the average number of clusters.

In terms of future research, two directions have been identified. First, the proposed bounded confidence models can be extended to discuss the fuzzy opinion formations, such as linguistic variable[20], intuitionistic fuzzy numbers[21], and hesitant fuzzy set $[22,23]$. Second, since the homogeneous bounded confidence is used to determine the confidence set, which limits the open-minded degree of some agents, in future research, generalizing the homogeneous bounded confidence into heterogeneous ones should be considered.

\section{References}

[1] M. Afshar, M. Asadpour. Opinion formation by informed agents. Journal of Artificial Societies and Social Simulation, 13, 2010.

[2] I. Palomares, F.J. Estrella, L. Martínez, F. Herrera. Consensus under a fuzzy context: Taxonomy, analysis framework AFRYCA and experimental case of study. Information Fusion, 20, 252-271, 2014.

[3] J. Kacprzyk, M. Fedrizzi. A 'soft' measure of consensus in the setting of partial (fuzzy) preferences. European Journal of Operational Research, 34, 316-325, 1988.

[4] E. Herrera-Viedma, F.J. Cabrerizo, J. Kacprzyk, W. Pedrycz. A review of soft consensus models in a fuzzy environment. Information Fusion, 17, 4-13, 2014.

[5] P. Krapivsky, S. Redner. Dynamics of majority rule in two-state interacting spin systems. Physical Review Letters, 90, 2003.

[6] M. Lewenstein, A. Nowak, B. Latane. Statistical mechanics of social impact. Physical Review A, 45, 763-776, 1992.

[7] G. Deffuant, D. Neau, F. Amblard, G. Weisbuch. Mixing beliefs among interacting agents. Advances in Complex Systems, 3: 87-98, 2000. 
[8] R. Hegselmann, U. Krause. Opinion dynamics and bounded confidence models, analysis, and simulation. Journal of Artificial Societies and Social Simulation, 5, 2000.

[9] R. Axelrod. The dissemination of culture: A model with local convergence and global polarization. Journal of Conflict Resolution, 41: 203226, 1997.

[10] D. Lim, H. Lee, H. Zo, A. Ciganek. Opinion formation in the digital divide. Journal of Artificial Societies and Social Simulation, 17, 2014.

[11] G. Weisbuch. Bounded confidence and social networks. The European Physical Journal BCondensed Matter and Complex Systems, 38: 339-343, 2004.

[12] D. Urbig, J. Lorenz, H. Herzberg. Opinion dynamics: The effect of the number of peers met at once. Journal of Artificial Societies and Social Simulation, 11, 2008.

[13] J. Lorenz. Continuous opinion dynamics with bounded confidence: A survey. International Journal of Modern Physics C, 18: 1839-1838, 2007.

[14] E. Kurmyshev, H.A. Juárez, R.A. GonzálezSilva. Dynamics of bounded confidence opinion in heterogeneous social networks: concord against partial antagonism. Physica A: Statistical Mechanics and its Applications, 390: 2945-2955, 2011.

[15] S. Fortunato, V. Latora, A. Pluchino, A. Rapisarda. Vector opinion dynamics in a bounded confidence consensus model. International Journal of Modern Physics C, 16: 1535-1551, 2005.

[16] S. Kurz, J. Rambau. On the HegselmannKrause conjecture in opinion dynamics. Journal of Difference Equations and Applications, 17: 859-876, 2011.

[17] Y. Shang. An agent based model for opinion dynamics with random confidence threshold. Communications in Nonlinear Science and $\mathrm{Nu}$ merical Simulation, 19: 3766-3777, 2014.

[18] Y.M. Wang, J.B. Yang, D.L. Xu. Interval weight generation approaches based on consistency test and interval comparison matrices. $A p$ plied Mathematics and Computation, 167: 252273, 2005.

[19] E. Conde, M. Pérez. A linear optimization problem to derive relative weights using an interval judgement matrix. European Journal of Operational Research, 201: 537-544, 2010.

[20] L.A. Zadeh. The concept of a linguistic variable and its application to approximate reasoning-I. Information Sciences, 8: 199-249, 1975.

[21] K.T. Atanassov. Intuitionistic fuzzy sets. Fuzzy Sets and Systems, 20: 87-96, 1986.

[22] V. Torra. Hesitant fuzzy sets. International Journal of Intelligent Systems, 25: 529-539, 2010.

[23] H.C. Liao, Z.S. Xu, X.J. Zeng, J.M. Merigó. Qualitative decision making with correlation co- efficients of hesitant fuzzy linguistic term sets. Knowledge-Based Systems, 76: 127-138, 2015. 